\title{
Detectors for Superboosted $\tau$-leptons at Future Circular Colliders
}

\section{Sourav Sen*, ${ }^{*}$ Ashutosh Kotwal, ${ }^{a, b}$ Sergei Chekanov, ${ }^{c}$ Lindsey Gray, ${ }^{b}$ Nhan Viet} Tran, ${ }^{b}$ and Shin-Shan $\mathbf{Y u}^{d}$

${ }^{a}$ Department of Physics, Duke University, Durham NC, USA

${ }^{b}$ Fermi National Accelerator Laboratory, Batavia IL, USA

${ }^{c}$ High Energy Physics Division, Argonne National Laboratory, Argonne IL, USA

${ }^{d}$ Department of Physics, National Central University, Chung-Li, Taiwan

\section{E-mail:}

sourav. sen@duke. edu, ashutosh. kotwal dduke.edu, chekanov@anl.gov,

lagrayefnal.gov,ntranefnal.gov, syuecern.ch

\begin{abstract}
We study the detector performance of $\tau$-lepton identification variables at very high energy proton colliders. We study hadronically-decaying $\tau$-leptons with transverse momentum in the TeV range. Calorimeters are benchmarked in various configurations in order to understand the impact of granularity and resolution on boosted $\tau$-lepton discrimination.
\end{abstract}

38th International Conference on High Energy Physics 3-10 August 2016

Chicago, USA

${ }^{*}$ Speaker. 


\section{Introduction}

Future experiments like FCC-hh (proton-proton collider at very high energy $\sqrt{s}=100 \mathrm{TeV}$ [1]) would probe Beyond Standard Model channels involving heavy resonances, many of which have $\tau$ leptons in their final states [2,3]. The detectors should therefore be able to detect the superboosted final states in order to reconstruct these heavy resonances. Additionally, the $\tau$ lepton is the most difficult charged lepton to identify, and it decays both hadronically $(\sim 65 \%)$ and leptonically ( $\sim 35 \%$ ) due to its heavy mass of $1.777 \mathrm{GeV}$. The hadronically decaying $\tau$ leptons (referred here as $\tau_{\text {had }}$ ) have QCD jets as their major background. A high-granularity silicon-tungsten calorimeter with sufficient radiation and interaction lengths to contain all the $\tau$-decay products has been designed. Since the granularity is very high, a fast simulation study would not be able to give the required insights into the detector design. Here we present the detector response to super-boosted $\tau_{\text {had }}$ jets $(\sim 500 \mathrm{GeV})$ using a full GEANT4-based simulation of the FCC-hh detector.

\section{Detector design}

As a starting point, we repurposed the basic design of the Silicon Detector ( $\mathrm{SiD})[4]$ of the International Linear Collider, because of its high solenoid field (5 Tesla) and the highly granular tracker and calorimeters. This made the detector silicon sensor-based, hence it is named "SiFCC". The Electromagnetic Calorimeter (ECAL) has 32 layers of tungsten absorber with $2 \mathrm{~cm} \times 2 \mathrm{~cm}$ silicon pixels, providing $\sim 35 X_{0}$ radiation lengths. In order to contain high $p_{T}$ jets, the Hadronic Calorimeter (HCAL) consists of 64 layers of steel absorber, giving 11.3 interaction lengths $\left(\lambda_{I}\right)$. The HCAL active material consists of $5 \mathrm{~cm} \times 5 \mathrm{~cm}$ scintillator pixels. The cylindrical barrel extends to pseudorapidity $|\eta|=1.7$. The total diameter of the detector is $19 \mathrm{~m}$.

\section{Event simulation and reconstruction}

For this study, $Z^{\prime}(1 \mathrm{TeV}) \rightarrow \tau \tau$ and $Z^{\prime}(1 \mathrm{TeV}) \rightarrow q \bar{q}$ Monte-Carlo events were used to model the signal and background processes respectively. The generator-level (referred to as truth-level) samples were obtained from a public repository for High Energy Physics Monte Carlo simulated events, HepSim [5], where they were produced using PYTHIA6/MADGRAPH8. These samples were processed through the SiFCC detector simulation. Owing to the di-jet topology of both signal and background events, the Durham Jet Algorithm was used to cluster the final-state particles into two jets. These jets are then passed on to the $\tau$-identification performance study.

\section{Identification of hadronically-decaying $\tau$-leptons}

$\tau$-leptons being color-neutral decay via the weak interaction. This inhibits quark \& gluon radiation in $\tau_{\text {had }}$ jets, distinguishing them from QCD jets as follows: (i) $\tau_{\text {had }}$ jets are more collimated and isolated; (ii) low track (charged-particle) multiplicity as $\tau$-leptons predominantly decay via 1-prong ( $\tau_{\text {had }} \rightarrow \pi^{ \pm} v_{\tau}+n \pi^{0}$, branching ratio: $\sim 21 \%$ ) or 3-prong ( $\tau_{\text {had }} \rightarrow 3 \pi^{ \pm} v_{\tau}+n \pi^{0}$, branching ratio: $\sim 70 \%$ ) channels; and (iii) presence of secondary vertex in the $\tau_{\text {had }}$ decay.

Based on these features, we use a set of variables to discriminate $\tau_{\text {had }}$ jets [6] against QCD background. We set the axis of a jet as the direction of the vector sum of momenta of its constituents. 
We divide the region containing the jet into two parts viz. the "core region" ( $\Delta R \leq 0.1$, where $\Delta R=\sqrt{(\Delta \eta)^{2}+(\Delta \phi)^{2}}$ about the jet axis) which contains $90 \%$ of the jet energy, and the "isolation region" $(0.1<\Delta R<0.4)$ which forms the periphery of the jet. The discriminating variables are:

Central energy fraction $\left(f_{\text {cent }}\right)$ Fraction of transverse energy deposited inside the region $\Delta R<$ 0.05 (i.e., half of the core radius) with respect to the total transverse energy deposited in the core region (Fig.1a).

Number of isolation tracks $\left(N_{\text {track }}^{\text {iso }}\right)$ Number of associated tracks in the isolation region (Fig.1b).

Leading-track momentum fraction $\left(f_{\text {track }}\right)$ Fraction of transverse momentum of the hardest associated track inside the core region with respect to the total transverse energy deposited in the core region.

Maximum $\Delta R\left(\Delta R_{\max }\right)$ Maximum $\Delta R$ between an associated track inside the core region and the jet axis.

Track radius $\left(R_{\text {track }}\right) \quad p_{T}$-weighted distance of the associated tracks within $\Delta R<0.4$ (i.e, core and isolation region combined) from the jet axis.

Track mass $\left(M_{\text {track }}\right)$ Invariant mass calculated using all the associated tracks within $\Delta R<0.4$, assuming $\pi^{ \pm}$mass for each track.

\section{Performance of $\tau$ discrimination variables in the SiFCC detector}

The discrimination variables worked well in capturing the salient features of $\tau_{\text {had }}$ jets at the truth-level as well as detector-level, as shown in Fig.1.

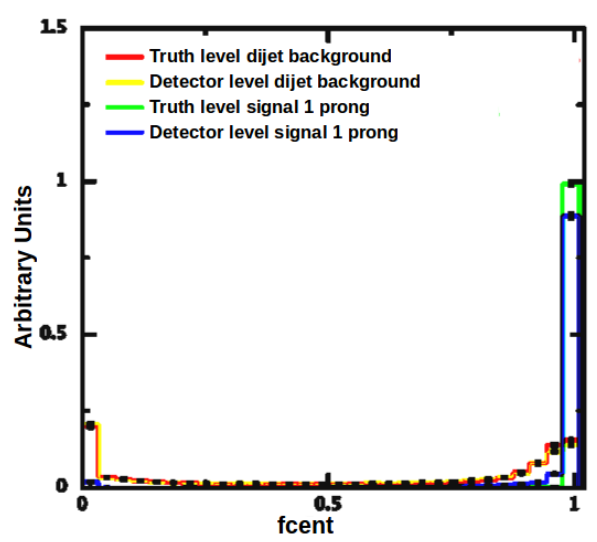

(a) $f_{\text {cent }}(1$ prong)

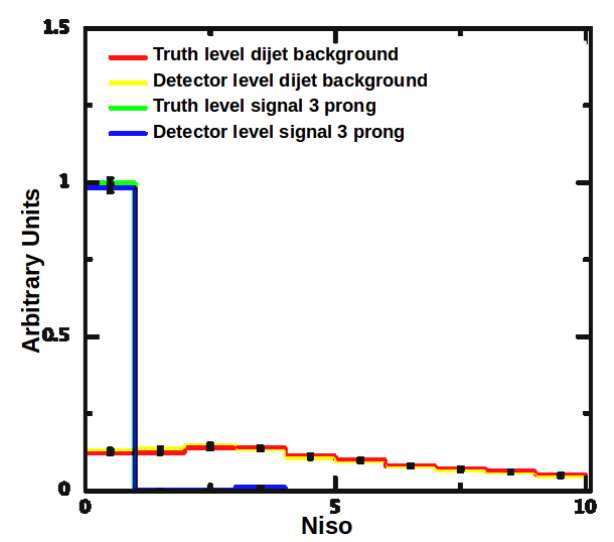

(b) $N_{\text {iso }}$ (3 prong)

Figure 1: Distributions of $\tau_{\text {had }}$ identification variables.

Due to sufficient granularity of the SiFCC detector design, the super-boosted final state particles could be resolved and reconstructed with high efficiency. This can be inferred from the exceptional agreement between the truth and detector-level distributions (Fig.1) of the discrimination variables for both signal and background. This high reconstruction efficiency also bolsters the 
performance of the discrimination variables at the detector level. The signal and background events are clearly separated and even a simple cut-based selection can give a high yield of signal events, with good background rejection. Thus, the discrimination variables are efficient in identification of hadronically-decaying $\tau$-leptons at the FCC-hh energy scale using the current detector design.

\section{Conclusion and further plans}

The good performance of super-boosted $\tau$-lepton identification provides an important physics benchmark for the granularity and size of the SiFCC detector. It also shows that the discrimination variables are applicable at the FCC-hh energy scales. For further optimization, we are planning to employ multivariate methods like Boosted Decision Tree (BDT) to improve super-boosted $\tau$-lepton identification at FCC-hh in the near future.

\section{Acknowledgements}

This research was performed using resources provided by the Open Science Grid, which is supported by the National Science Foundation and the U.S. Department of Energy's Office of Science. Argonne National Laboratory's work was supported by the U.S. Department of Energy, Office of Science under contract DE-AC02-06CH11357. The Fermi National Accelerator Laboratory (Fermilab) is operated by Fermi Research Alliance, LLC under Contract No. DE-AC02-07CH11359 with the United States Department of Energy.

\section{References}

[1] Benedikt, Michael. "Future Circular Collider (FCC) study-status." Proceedings of 1st Future Hadron Collier Workshop, CERN, Geneva Switzerland. 2014.

[2] Kotwal, A. V., S. Chekanov, and M. Low. "Double Higgs boson production in the $4 \tau$ channel from resonances in longitudinal vector boson scattering at a $100 \mathrm{TeV}$ collider." Physical Review D 91.11 (2015): 114018.

[3] Kotwal, Ashutosh V., et al. "Singlet-Catalyzed Electroweak Phase Transitions in the $100 \mathrm{TeV}$ Frontier." Physical Review D 94.03 (2016): 035022.

[4] Behnke, Ties, et al. "The International Linear Collider Technical Design Report-Volume 4: Detectors." arXiv preprint arXiv:1306.6329 (2013).

[5] Chekanov, S. V. "HepSim: a repository with predictions for high-energy physics experiments." Advances in High Energy Physics 2015 (2015).

[6] Aad, Georges, et al. "Identification and energy calibration of hadronically-decaying $\tau$ leptons with the ATLAS experiment in $p p$ collisions at $\sqrt{s}=8 \mathrm{TeV}$." The European Physical Journal C 75.7 (2015): $1-33$. 